\title{
Site-Selective Halogenation of Polyoxovanadate Clusters: Atomically Precise Models for Electronic Effects of Anion Doping in $\mathrm{VO}_{2}$
}

Brittney E. Petel, ${ }^{1+}$ Rachel L. Meyer, ${ }^{1 \ddagger}$ Michela L. Maiola, ${ }^{1}$ William W. Brennessel, ${ }^{1}$ Astrid M. Müller, ${ }^{* 2}$

Ellen M. Matson*1

${ }^{1}$ Department of Chemistry, University of Rochester, Rochester, New York 14627, United States

${ }^{2}$ Department of Chemical Engineering, University of Rochester, Rochester, New York 14627, United States

Denotes authors contributed equally.

Corresponding author emails: astrid.mueller@rochester.edu, matson@chem.rochester.edu

\section{Supporting Information Table of Contents:}

Figure S1. ${ }^{1} \mathrm{H}$ NMR Spectrum of $\mathbf{1}-\mathbf{V}_{6} \mathbf{O}_{7}{ }^{-2}$ and $\mathbf{3}-\mathbf{V}_{6} \mathbf{O}_{6} \mathbf{C l}^{-\mathbf{1}}$

Figure S2. Cyclic Voltammogram of crude reaction mixture following the addition of two equivalents of $\mathrm{AlCl}_{3}$ to complex $\mathbf{2}-\mathbf{V}_{6} \mathbf{O}_{7}^{-2}$. S2

Figure S3. ESI-MS (-)ve of 3-V $6 \mathrm{O}_{6} \mathrm{Cl}^{-1}$ .S3

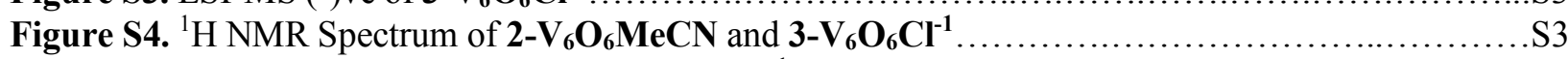

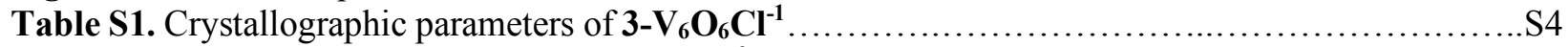

Table S2. Crystallographic parameters of $1-\mathrm{V}_{6} \mathrm{O}_{7}{ }^{-2}$.... .S5 
Figure S1. ${ }^{1} \mathrm{H}$ NMR spectra of $\mathbf{1}-\mathbf{V}_{6} \mathbf{O}_{7}^{-\mathbf{2}}$ (bottom, red) and $\mathbf{3 - V _ { 6 }} \mathbf{O}_{6} \mathbf{C l}^{-\mathbf{1}}$ (top, blue) in $\mathrm{CDCl}_{3}$.

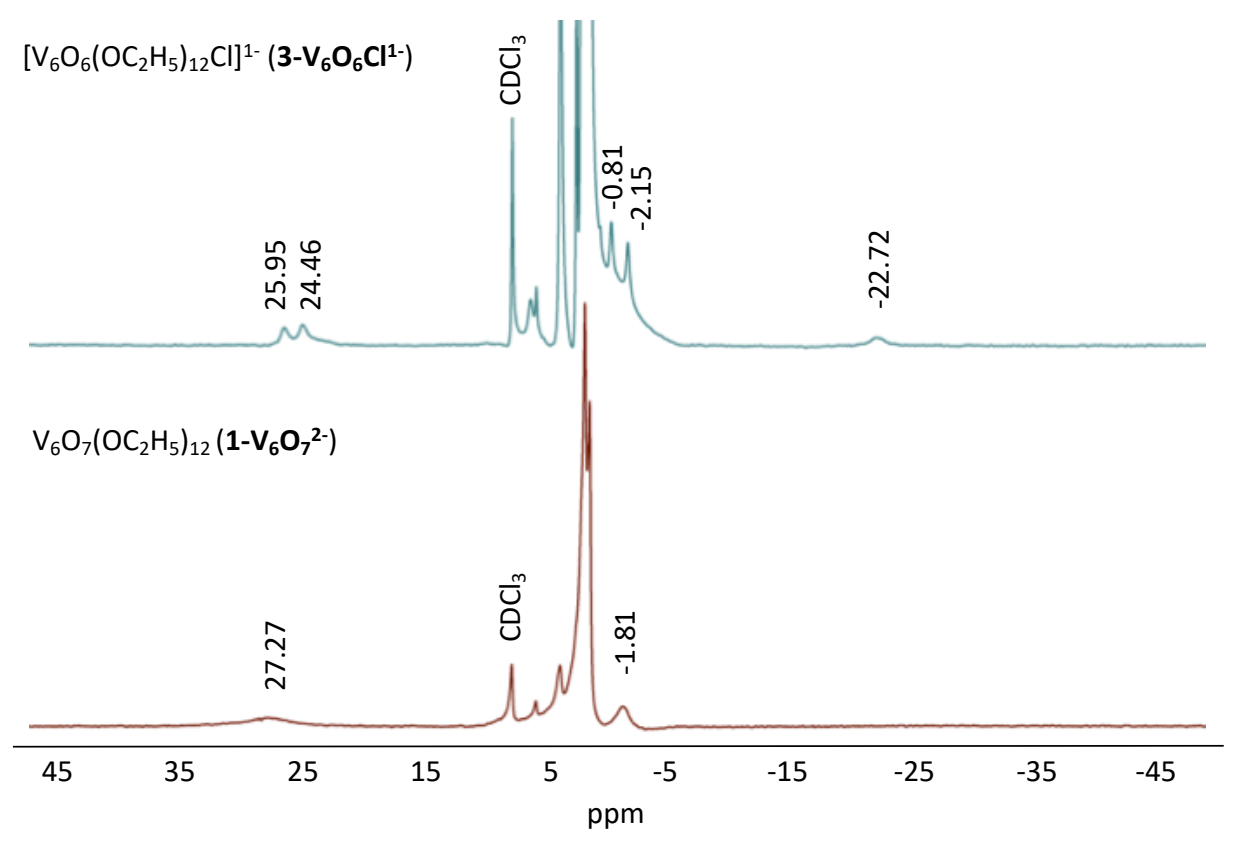

Figure S2. Cyclic voltammogram of the crude product following addition of two equivalents of $\mathrm{AlCl}_{3}$ to $\mathbf{1 - V _ { 6 }} \mathbf{O}_{7}{ }^{-2}$. The $\mathrm{CV}$ was collected in dichloromethane with $0.1 \mathrm{M}\left[{ }^{\mathrm{n}} \mathrm{Bu}_{4} \mathrm{~N}\right]\left[\mathrm{PF}_{6}\right]$ as the supporting electrolyte.

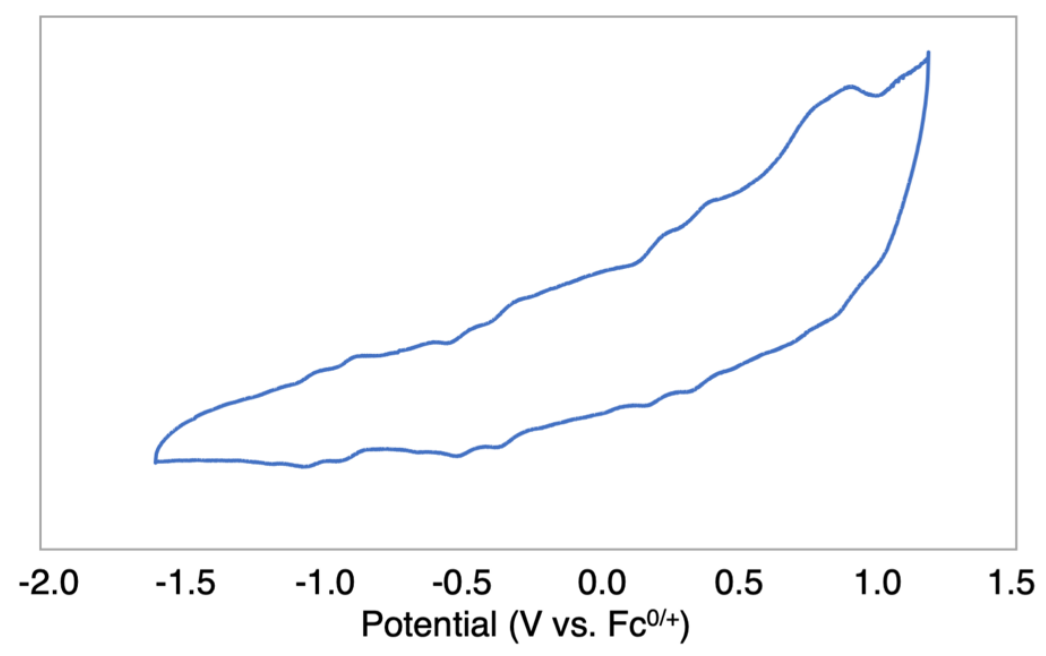


Figure S3. ESI-MS (-)ve of $\mathbf{3}-\mathbf{V}_{6} \mathbf{O}_{6} \mathbf{C l}^{-\mathbf{1}}\left(\mathrm{m} / z=977 \mathrm{amu} ;\left[\mathrm{V}_{6} \mathrm{O}_{6} \mathrm{Cl}\left(\mathrm{OC}_{2} \mathrm{H}_{5}\right)_{12}\right]^{1-}\right)$.

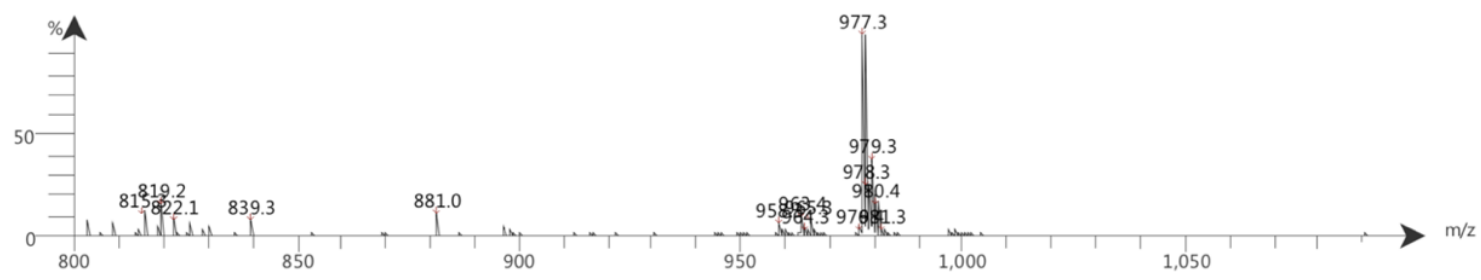

Raw ESI-MS Data
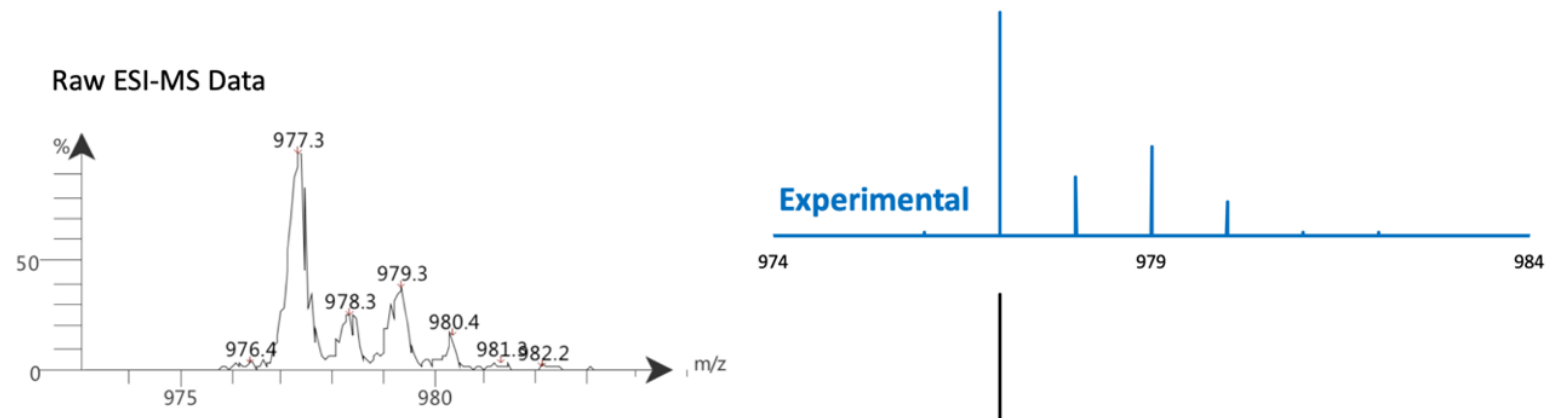

Figure S4. ${ }^{1} \mathrm{H}$ NMR spectra of $2-\mathbf{V}_{6} \mathrm{O}_{6} \mathrm{MeCN}$ (bottom, red) and $3-\mathbf{V}_{6} \mathbf{O}_{6} \mathrm{Cl}^{-\mathbf{1}}$ (top, blue) in $\mathrm{CDCl}_{3}$.

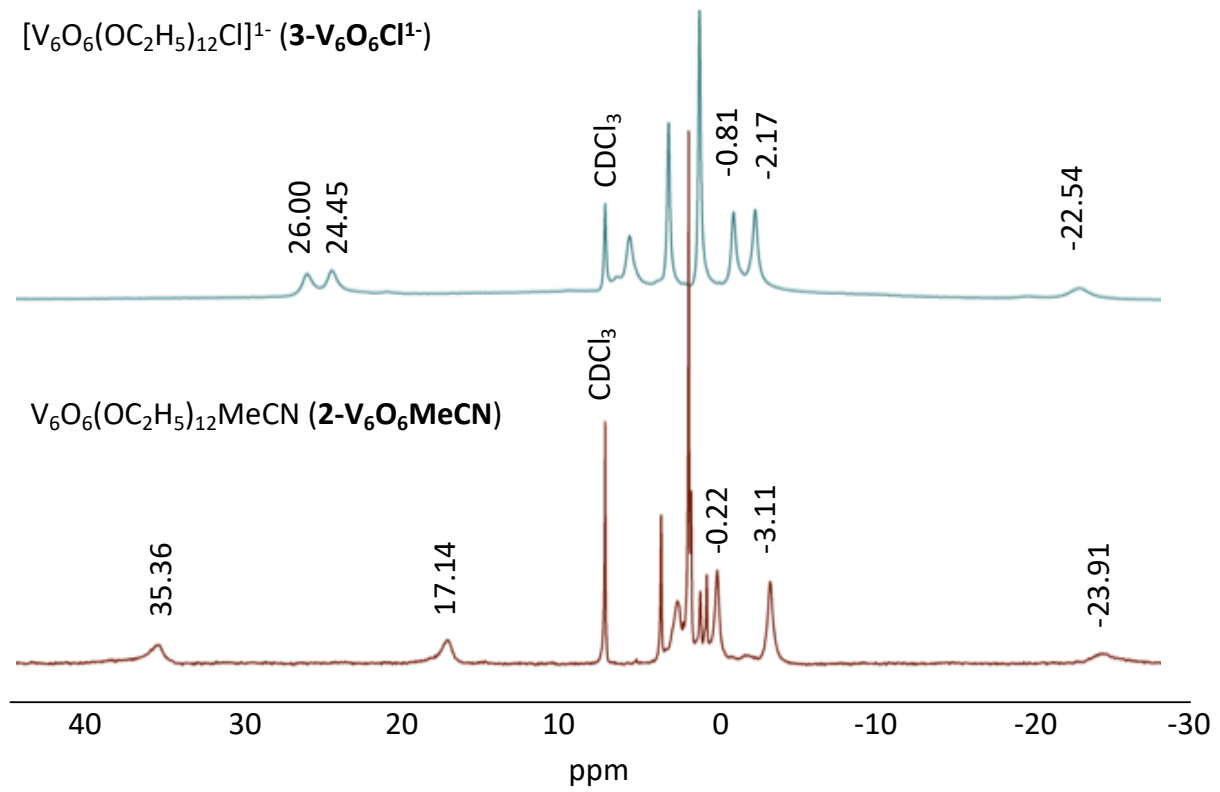


Table S1. Crystallographic Parameters of $\mathbf{3 - V _ { 6 }} \mathbf{O}_{6} \mathbf{C l}^{\mathbf{1}}$ (CCDC: 1954473).

\begin{tabular}{|c|c|c|}
\hline Empirical formula & \multicolumn{2}{|l|}{$\mathrm{C}_{32} \mathrm{H}_{80} \mathrm{ClO}_{18} \mathrm{~V}_{6}$} \\
\hline Formula weight & \multicolumn{2}{|l|}{1108.06} \\
\hline Temperature & \multicolumn{2}{|l|}{$100.0(4) \mathrm{K}$} \\
\hline Wavelength & \multicolumn{2}{|l|}{$1.54184 \AA$} \\
\hline Crystal system & \multicolumn{2}{|l|}{ Monoclinic } \\
\hline Space group & \multicolumn{2}{|l|}{$I 2 / a$} \\
\hline Unit cell dimensions & $\begin{array}{l}\mathrm{a}=20.9220(4) \AA \\
\mathrm{b}=11.57810(10) \AA \\
\mathrm{c}=23.8929(4) \AA\end{array}$ & $\begin{array}{l}\alpha=90^{\circ} \\
\beta=122.394(3)^{\circ} \\
\gamma=90^{\circ}\end{array}$ \\
\hline Volume & \multicolumn{2}{|l|}{$4887.08(19) \AA^{3}$} \\
\hline Z & \multicolumn{2}{|l|}{4} \\
\hline Reflections collected & \multicolumn{2}{|l|}{21222} \\
\hline Independent reflections & \multicolumn{2}{|l|}{5124} \\
\hline Goodness-of-fit on F2 & \multicolumn{2}{|l|}{1.057} \\
\hline $\begin{array}{l}\text { Final R indices } \\
{[\mathrm{I}>2 \operatorname{sigma}(\mathrm{I})]}\end{array}$ & \multicolumn{2}{|c|}{$\mathrm{R} 1=0.0436, \mathrm{wR} 2=0.1224$} \\
\hline
\end{tabular}


Table S2. Crystallographic Parameters of $\mathbf{1}-\mathbf{V}_{\mathbf{6}} \mathbf{O}_{7}^{-\mathbf{2}}$ (CCDC: 1954477).

\begin{tabular}{|c|c|c|}
\hline Empirical formula & \multicolumn{2}{|l|}{$\mathrm{C}_{56} \mathrm{H}_{132} \mathrm{~N}_{2} \mathrm{O}_{19} \mathrm{~V}_{6}$} \\
\hline Formula weight & \multicolumn{2}{|l|}{1443.27} \\
\hline Temperature & \multicolumn{2}{|l|}{$100.00(10) \mathrm{K}$} \\
\hline Wavelength & \multicolumn{2}{|l|}{$1.54184 \AA$} \\
\hline Crystal system & \multicolumn{2}{|l|}{ orthorhombic } \\
\hline Space group & \multicolumn{2}{|l|}{$P b c a$} \\
\hline Unit cell dimensions & $\begin{array}{l}\mathrm{a}=19.0957(3) \AA \\
\mathrm{b}=18.7627(3) \AA \\
\mathrm{c}=20.0038(3) \AA\end{array}$ & $\begin{array}{l}\alpha=90^{\circ} \\
\beta=90^{\circ} \\
\gamma=90^{\circ}\end{array}$ \\
\hline Volume & \multicolumn{2}{|l|}{$7167.10(19) \AA^{3}$} \\
\hline Z & \multicolumn{2}{|l|}{4} \\
\hline Reflections collected & \multicolumn{2}{|l|}{38510} \\
\hline Independent reflections & \multicolumn{2}{|l|}{7516} \\
\hline Goodness-of-fit on F2 & \multicolumn{2}{|l|}{1.076} \\
\hline $\begin{array}{l}\text { Final } R \text { indices } \\
{[\mathrm{I}>2 \operatorname{sigma}(\mathrm{I})]}\end{array}$ & \multicolumn{2}{|c|}{$\mathrm{R} 1=0.0352, \mathrm{wR} 2=0.0931$} \\
\hline
\end{tabular}

